\section{JTI}

JOURNAL OF

TRAUMA AND INJURY

\title{
Traumatic Posterior Fossa Subdural Hematoma in a Neonate: A Case Report
}

\author{
Ki Seong Eom, M.D., Ph.D. \\ Department of Neurosurgery, Wonkwang University School of Medicine, Iksan, Korea
}

Received: March 9, 2020

Revised: April 5, 2020

Accepted: April 7, 2020

\section{Correspondence to}

Ki Seong Eom, M.D., Ph.D.

Department of Neurosurgery, Wonkwang University Hospital, 895 Muwang-ro, Iksan

54538 , Korea

Tel: +82-63-859-1462

Fax: +82-63-852-2606.

E-mail:kseom@wonkwang.ac.kr
Although traumatic posterior fossa subdural hematoma (TPFSH) in neonates immediately after birth is extremely rare, it can pose a serious clinical problem in the neonatal period. Here, the author presents the case of a 3-day-old male infant who underwent emergency surgical treatment of TPFSH with a favorable outcome. Debate continues about surgical versus conservative treatment of TPFSH in neonates. The clinical symptoms, extent of hemorrhage, early diagnosis, and prompt and appropriate surgery are the most important factors in the treatment of TPFSH in neonates. Therefore, neurosurgeons should establish treatment strategies based on the newborn's clinical condition, the size and location of the TPFSH, and the potential of the hematoma to cause long-term complications.

Keywords: Trauma; Hematoma, subdural; Neonate

\section{INTRODUCTION}

Traumatic posterior fossa subdural hematoma (TPFSH) in neonates immediately after birth is extremely rare. It is associated with delayed delivery, breech presentation, vacuum or forceps use during delivery, and primiparity or excessive multiparity [1-5]. TPFSH mainly occurs in babies delivered at full term. Typically, neurological abnormalities and symptoms develop after an initial asymptomatic period of 12 hours to 4 days [4]. Since the first successful surgical treatment was reported by Coblentz in 1940 [6], the development of diagnostic neuroimaging techniques, such as cranial ultrasonography, computed tomography (CT), and magnetic resonance imaging (MRI) have enabled the early diagnosis of acute TPFSH in neonates [1]. Surgical or conservative treatment may be used. The author presents a case of a favorable outcome after emergency surgical treatment of TPFSH in a neonate.

This is an Open Access article distributed under the terms of the Creative Commons Attribution Non-Commercial License (http://creativecommons.org/licenses/by-nc/4.0/) which permits unrestricted noncommercial use, distribution, and reproduction in any medium, provided the original work is properly cited. 


\section{CASE REPORT}

A 3-day-old male infant was admitted with intermittent vomiting, one seizure, and a large head size. The baby was born after a normal labor at 39 weeks of gestation. His birth weight was $3.2 \mathrm{~kg}$, and his Apgar scores were 8 and 9 . His family history was unremarkable, and physical examination revealed an abnormally large head circumference $(40.5 \mathrm{~cm})$, a bulging fontanelle, and bilateral scalp swelling in the parietal area. The patient was unable to suck well, and intermittent bradycardia was observed. A neurological examination revealed that overall muscle tone was slightly decreased. Laboratory studies, including co- agulation, were normal. Cranial ultrasonography showed dilation of both lateral ventricles (Fig. 1A). Cranial CT revealed acute subdural hematoma in the posterior fossa that was up to $17 \mathrm{~cm}$ thick, resulting in acute obstructive hydrocephalus and separation of the skull suture lines (Fig. 1B). The patient underwent bilateral suboccipital craniectomy and removal of hematoma in the prone position. Tearing of the cortical bridging veins was identified as the cause of the hematoma. The operation lasted 2 hours. The patient's vital signs remained stable throughout the operation, and no specific adverse events took place. Repeat cranial ultrasonography scans obtained 1 day after the operation revealed that the size of both lat-
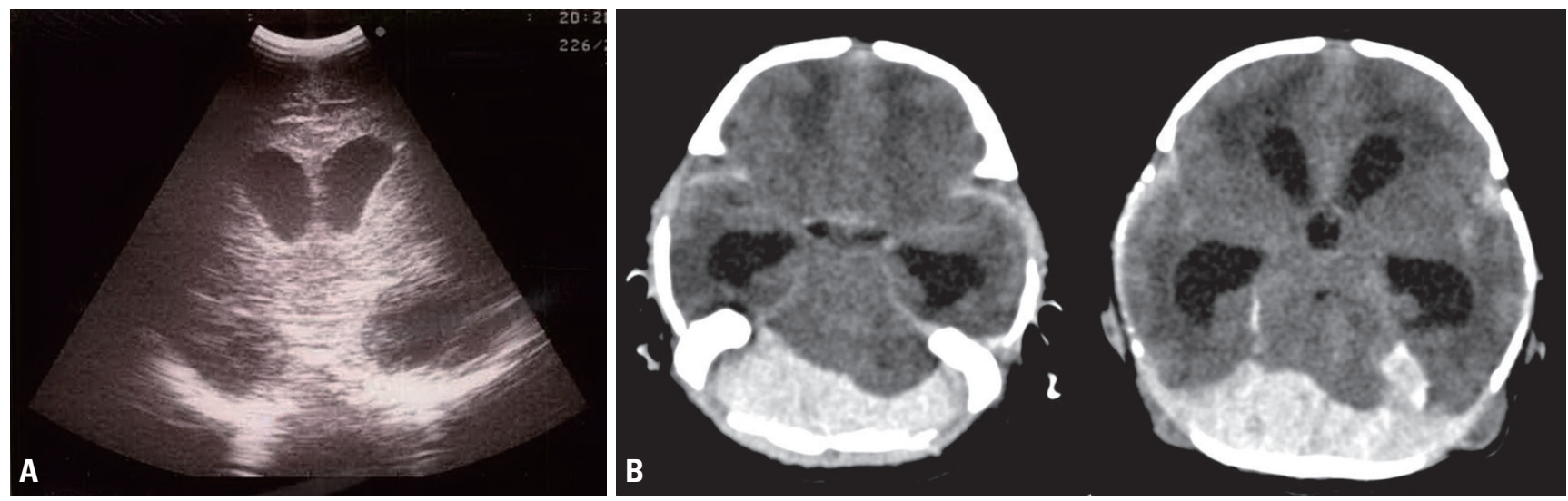

Fig. 1. (A) Cranial ultrasonography revealing dilation of both lateral ventricles. (B) Cranial computed tomography revealed an acute subdural hematoma in the posterior fossa that was up to $17 \mathrm{~cm}$ thick, resulting in acute obstructive hydrocephalus and separation of the skull suture lines.
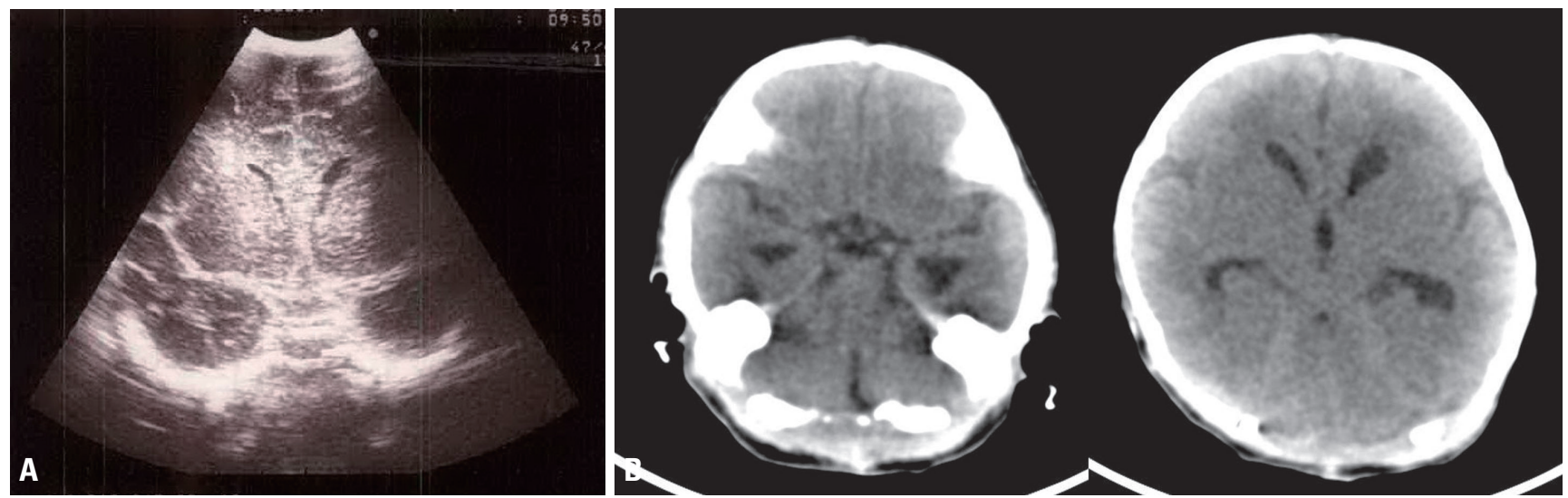

Fig. 2. (A) Repeat cranial ultrasonography scans obtained 1 day after the operation showing that the size of both lateral ventricles had decreased to normal. (B) Repeat cranial computed tomography scans obtained 2 days after the operation revealing that the hematoma had been almost completely removed. The size of the ventricles and the width of the separated skull sutures were restored to normal. 
eral ventricles had decreased to normal (Fig. 2A). Repeat cranial CT scans obtained 2 days postoperatively revealed that the hematoma had been almost completely removed (Fig. 2B). The size of the ventricles and the width of the separated skull sutures were restored to normal. The postoperative course was uneventful, and the patient was discharged after 14 days with no neurological deficits.

\section{DISCUSSION}

TPFSH in newborn babies is a traumatic complication at birth. Although TPFSH is a very rare condition, it is clinically critical, and is most easily diagnosed by non-contrast CT [1]. The size of the TPFSH can range from a large hematoma with significant mass effects to a small, clinically insignificant hematoma that can only be detected by CT. Hematoma encroaching into the limited space of the posterior fossa can cause neurological abnormalities. Typical symptoms and signs include tension or bulging of the fontanelle, increased head circumference, apnea, bradycardia, vomiting, loss of the sucking or Moro reflex, cranial nerve palsy, seizure, and opisthotonos [4,7].

Three main mechanisms lead to TPFSH in newborn babies. The first involves pressure on the occiput during the birth process. Hemsath [8] analyzed 32 cases at autopsy and suggested that as the fetal head passed through the birth canal, the occipital bone was pressed excessively and thus injured by the mother's pubic bone, resulting in TPFSH. Although this mechanism could explain direct cerebellar trauma, compression, and death of stillborn babies, it was insufficient to explain bleeding in the subdural or subtentorial area [7]. In addition, dislocation of the occipital bone is an extremely rare finding in neonates. The second mechanism is excessive vertical molding during delivery. When severe pressure is applied to the anterior-posterior axis of the fetal head, or fronto-occipital elongation of the skull occurs, the pressure is transmitted to the falx and tentorium. This transmitted pressure causes tears or ruptures in large veins, such as the vein of Galen or straight sinus, resulting in TPFSH [9]. Hemorrhage resulting from damage to the vein of Galen or straight sinus may occur in a deep portion of the intracranial cavity, and simultaneously in the upper and lower compartments of the tentorium. This can cause serious neurological deterioration or death resulting from impaired drainage of the deep venous system. The third mechanism is traumatic injury of the bridging veins on the tentorial surface of the cerebellum or tentorial leaflet. Perrin et al. [7] confirmed tearing of the transverse sinus or tentorium, as well as bleeding of the cerebellar bridging vein, in five of eight patients with TPFSH who underwent surgery. They speculated that in most of the other newborns with TPFSH, there may have been damage to the cerebellar bridging vein, the tentorial leaflet orthodontic vein, or the tentorial leaflet. In this case, tearing of the cortical bridging veins was identified as the cause of hematoma. Therefore, it was thought that the main mechanism of TPFSH in this neonate was tearing of the bridging veins due to mechanical compression and distortion of the head during the birth process.

Due to advances in imaging techniques, it is now possible to diagnose TPFSH in the neonatal period more easily and rapidly. The advantage of cranial CT is that it enables accurate localization of the hematoma in both the coronal and sagittal planes. However, MRI can identify the anatomical structures of the posterior fossa better than CT. In particular, sagittal imaging by MRI can help determine the size and location of the hematoma associated with the tentorium, and identify the degree of compression of the brainstem or cerebral aqueduct [1]. Ultrasonography of the posterior fossa is relatively difficult; therefore, making an early diagnosis using this modality is challenging, and misdiagnosis is likely. However, ultrasonography is helpful in the follow-up period to track the absorption of hematoma and the development of hydrocephalus after the diagnosis of TPFSH. Other important findings include blood-tinged cerebrospinal fluid, decreased hemoglobin concentration, and retinal hemorrhage. Electroencephalography is usually normal [4].

Debate continues about surgical versus conservative treatment of TPFSH in neonates. Although some recommend conservative treatment (despite the common complication of hydrocephalus), most studies have reported better results with surgical treatment $[4,7]$. Neonates with small hematomas that do not cause circulatory disturbances in the cerebrospinal fluid or brainstem compression are more likely to recover spontaneously, 
and therefore only conservative treatment is needed. The use of acetazolamide or furosemide may be helpful as part of conservative treatment [9]. However, neonates with progressive brainstem compression due to a large hematoma can die without immediate surgical treatment. Govaert et al. [10] suggested that surgical treatment should be performed to prevent brainstem compression or obstructive hydrocephalus in infants with 1) clinical signs of life-threatening brainstem compression such as apnea, bradycardia, and hypotension; 2) acute obstructive hydrocephalus with increased intracranial pressure; or 3) a hematoma in the posterior fossa larger than $15 \mathrm{~cm}$. Several surgical procedures have been used in neonates with clinically critical TPFSH, including subdural punctures through the lambdoid suture and squamous portion of the occipital bone, burr drainage of the hematoma, extraventricular drainage for obstructive hydrocephalus, and craniectomy with removal of the hematoma [1].

In conclusion, there is no doubt that the clinical symptoms, extent of hemorrhage, early diagnosis, and prompt and appropriate surgery are the most important factors in the treatment of TPFSH in neonates. Therefore, neurosurgeons should establish treatment strategies based on the clinical condition of the newborn, the size and location of the TPFSH, and the potential of the hematoma to cause long-term complications. Further research is warranted to find ways of avoiding this rare birth injury.

\section{REFERENCES}

1. Blauwblomme T, Garnett M, Vergnaud E, et al. The management of birth-related posterior fossa hematomas in neonates. Neurosurgery 2013;72:755-62.

2. Hanigan WC, Morgan AM, Stahlberg LK, Hiller JL. Tentorial hemorrhage associated with vacuum extraction. Pediatrics 1990;85:534-9.

3. Volpe JJ. Neonatal intracranial hemorrhage. Pathophysiology, neuropathology, and clinical features. Clin Perinatol 1977;4:77102.

4. von Gontard A, Arnold D, Adis B. Posterior fossa hemorrhage in the newborn--diagnosis and management. Pediatr Radiol 1988;18:347-8.

5. Welch K, Strand R. Traumatic parturitional intracranial hemorrhage. Dev Med Child Neurol 1986;28:156-64.

6. Coblentz RG. Cerebellar subdural hematoma in infant 2 weeks old with secondary hydrocephalus: operation with recovery. Surgery 1940;8:771-6.

7. Perrin RG, Rutka JT, Drake JM, et al. Management and outcomes of posterior fossa subdural hematomas in neonates. Neurosurgery 1997;40:1190-200.

8. Hemsath FA. Birth injury of the occipital bone, with a report of thirty-two cases. Am J Obstet Gynecol 1934;27:194-203.

9. Shinnar S, Gammon K, Bergman EW Jr, Epstein M, Freeman JM. Management of hydrocephalus in infancy: use of acetazolamide and furosemide to avoid cerebrospinal fluid shunts. J Pediatr 1985;107:31-7.

10. Govaert P, Calliauw L, Vanhaesebrouck P, Martens F, Barrilari A. On the management of neonatal tentorial damage. Eight case reports and a review of the literature. Acta Neurochir (Wien) 1990;106:52-64. 